MODULAR ARCHITECTURE

CASE ANALYSIS:

THE ROTTERDAM'S

CUBE HOUSES,

NETHERLANDS

KEYWORDS

Industrialization; innovation; modulation.

\section{ABSTRACT}

This work addresses the analysis of a particular case of Industrialized Modular Architecture: the Cube Houses or Kubuswoningen, a housing complex located in the city of Rotterdam, Netherlands. This analysis delves into the conception, development and materialization of the aforementioned complex, the reasons why it is located within the Modular Architecture, the application of the geometric principles of the Polychube Theory and how the results we arrive at can help us to generate a new and optimized way of projecting and building in architecture.
ANÁLISE DE CASO DE ARQUITETURA MODULAR: AS CASAS CUBO EM ROTTERDAM. HOLANDA

\section{RESUMO}

Industrialização; inovação; modulação.

\section{PALAURAS CHAUE}

Este trabalho aborda a análise de um caso particular de Arquitetura Modular Industrializada: as Casas Cubo ou Kubuswoningen, um conjunto habitacional situado na cidade de Rotterdam, Holanda. Esta análise aprofunda-se sobre a concepção desenvolvimento e materialização do referido complexo, as razões pelas quais está inserido na Arquitetura Modular, a aplicação dos princípios geométricos da Teoria do Policubo e como os resultados a que chegamos podem nos ajudar a gerar uma nova e otimizada forma de projetar e construir em arquitetura. 


\title{
ANÁLISIS DE CASO DE ARQUITECTURA MODULAR: LAS KUBUUSWONINGEN O CASAS CUBO EN ROTTERDAM, HOLANDA
}

\author{
Maira L. DOUTHAT', Mauricio M. GAUNA ${ }^{2}$ \\ Lorena B. LONGA ${ }^{3}$, Rosanna G. MORAN ${ }^{4}$ \\ mairadouthat@gmail.com
}

\begin{abstract}
Adscriptos (1, 2 y 3) y JTP (4) en Construcciones II A, ITDAHu (Instituto de Investigaciones Tecnológicas para el Diseño Ambiental del Hábitat Humano), Facultad de Arquitectura y Urbanismo de la UNNE.
\end{abstract}

PALABRAS CLAUE

Industrialización; innovación; modulación.

RESUMEN

Este trabajo aborda el análisis de un caso particular de Arquitectura Modular Industrializada: Ias Casas Cubo o Kubuswoningen, complejo habitacional situado en la ciudad de Rotterdam, Holanda. En dicho análisis se profundiza sobre la concepción, desarrollo y materialización del mencionado complejo, las razones por las cuales se lo ubica dentro de la Arquitectura Modular, la aplicación de los principios geométricos de la Teoría de los Policubos y cómo los resultados a los que arribemos pueden ayudarnos a generar un nueva y optimizada forma de proyectar y construir en arquitectura. 


\section{OBJETIUO}

Analizar la referida obra de Piet Blom, con el empleo de diversas metodologías relacionadas entre sí como una manera diferente de concebir y desarrollar el proceso creativo, tomando como base las ventajas de esta forma no convencional de hacer arquitectura que se utilizó en la ciudad de Róterdam. Esto motiva un interés de plantear el desafío de explorar los innovadores modos de construir y considerar el hábitat humano, buscando nuevas alternativas que sirvan de muestra a los estudiantes de Construcciones II A, de forma que puedan rescatar lo positivo en el proceso creativo de los trabajos en la asignatura.

\section{INTRODUCCIÓN}

El presente artículo surge de la investigación iniciada al comienzo del ciclo lectivo 2019 como parte de nuestro trabajo de adscripción para la cátedra Construcciones II A de la carrera de Arquitectura de la Facultad de Arquitectura y Urbanismo, de la Universidad Nacional del Nordeste. La asignatura Construcciones II A tiene como objetivo el estudio de los diferentes sistemas constructivos racionalizados e industrializados. Es en este marco en el cual se aborda el análisis e investigación de una obra de arquitectura paradigmática: las Kubuswoningen o Casas Cubo del arquitecto holandés Piet Blom, escogida por presentar una trama inusual e innovadora en la configuración del complejo habitacional y por tratarse de un sistema de construcción no tradicional, identificando en ella principios básicos de la industria, como la racionalización del trabajo, la normalización constructiva y la tipificación productiva.

Es particularmente interesante esta obra, ya que surge de los requerimientos de dar respuesta a diferentes problemáticas que se presentan en el sitio donde está implantada, respuestas que se han visto satisfechas mediante la implementación

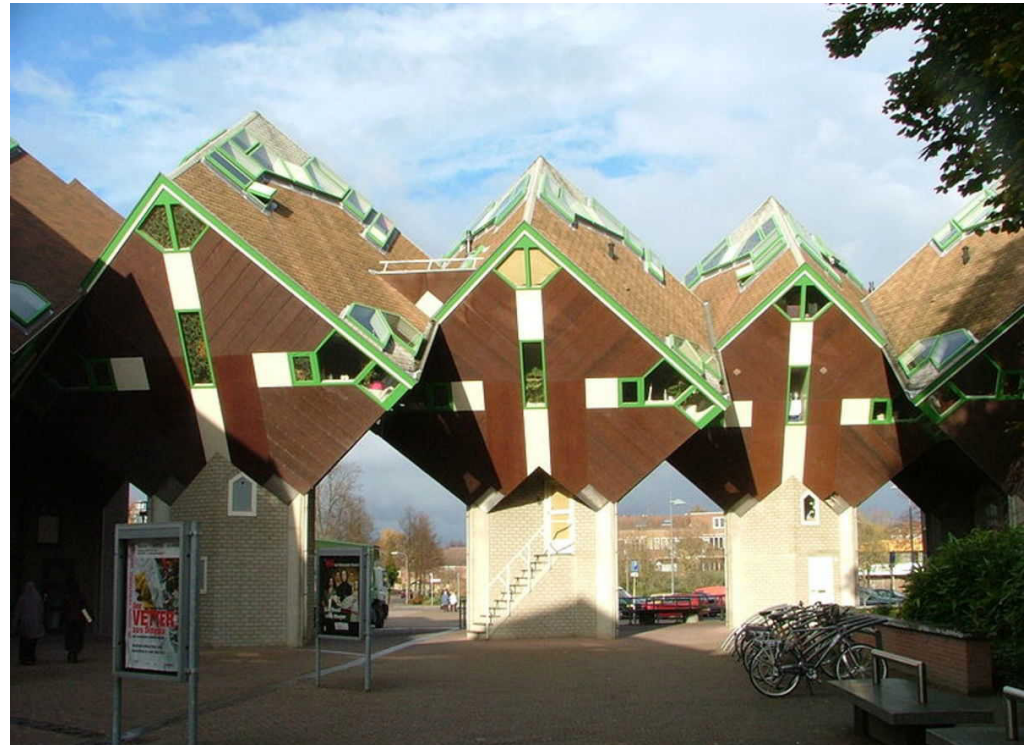

Figura 1. Casas cubo en Helmond, Países Bajos. Fuente: https:// planergo.com/es/sitios/Casas-cubo-de-Helmond/

de soluciones no convencionales, que optimizaron y revalorizaron la identidad del lugar. Podemos relacionar a su vez su concepción con criterios de la Arquitectura Modular (AM), consistente en el diseño y manejo de sistemas compuestos por elementos constructivos industrializados, generados con dimensiones modulares coordinadas, con capacidad de conectarse entre sí y ser ubicados en diferentes posiciones y situaciones en obra, ser reemplazados o intercambiados, sin que estas operaciones afecten el sistema ni provoquen una significativa merma en los estándares de calidad del producto final diseñado.

Para finalizar, aplicaremos a esta unidad de estudio los principios básicos de un concepto compatible con la Arquitectura Modular denominado Teoría de los Policubos que, mediante la manipulación de estos brinda las bases para el desarrollo de diferentes habilidades creativas y visuales, permitiéndonos comprender de manera clara la relación intrínseca entre ambos conceptos y cómo pueden ayudarnos a visualizar una nueva manera de pensar y construir Arquitectura, para nuestro beneficio y el del medio donde vivimos.

\section{DESARROLLO}

Cada vez estamos más acostumbrados a toparnos con los llamados "experimentos arquitectónicos", pero aunque parezca algo contemporáneo y novedoso, nada más lejos de la realidad. Desde los comienzos de la arquitectura, cada diseñador está llamado a la innovación, a resaltar sus obras sobre los convencionalismos típicos de lo urbano, a velar por permanecer en el recuerdo de cualquier ciudadano de a pie (JM3 Studio, 2015).

\section{Kubuswoningen: un bosque urbano singular en la ciudad de Rotterdam}

Las Casas Cubo -o Kubuswoningen en su idioma original- son una obra del arquitecto holandés Piet Blom (8 de febrero de 1934, Ámsterdam - 8 de junio de 1999, Dinamarca), representante del Estructuralismo Arquitectónico en Holanda (nl.wikipedia.org, 2019). Empleando una alta densidad de construcción, combinando funciones y la creación de diferentes capas, Blom se esfuerza por crear un entorno urbano residencial animado y agradable. Hasta su muerte en 1999, diseñó casas y urbanizacio- 
nes para rechazar la severa frialdad de posguerra, siempre buscando la luz de una arquitectura más cálida y humana. Sus dibujos, diagramas $y$ hogares retratan un compromiso afectuoso para reconciliar los elementos de la cultura con la arquitectura que nos rodea. Caracterizado por su uso de colores vivos y geometrías arquitectónicas igualmente expresivas, proyectos como la "Kasbah" y las Casas Cubo en Rotterdam son testimonio de su creencia de que la arquitectura sirve a la gente, y no al revés (circarq.wordpress.com, 2018) En 1971, Piet Blom recibió el encargo de estudiar el área del Oude Haven (Puerto Viejo) de Rotterdam, Holanda. El objetivo era revitalizar la zona, que permanecía sin explotar desde la Segunda Guerra Mundial y generar espacios residenciales dando prioridad a la vivienda social, los proyectos recreativos y la arquitectura "lúdica". Pocos edificios sobrevivieron al bombardeo de 1940, y como resultado del cambio de gobierno en 1974, la regeneración urbana y la vivienda se convirtieron en las principales prioridades para el concejo municipal.

Hans Mentink, el político encargado de la ordenación del territorio en e momento, se había cansado de la arquitectura utilitaria, e impresionado por casas cubo de Blom en Helmond (figura 1) le adjudicó el contrato para crear un plan de desarrollo para el área de Oude Haven.

El proyecto inicial contemplaba 74 casas cubo y un centro cultural. Finalmente, Blom dividió el desarrollo de la zona en tres proyectos: el Blaaktoren, una torre de departamentos cuya forma asemeja a la de un lápiz, que se puede observar al fondo a la izquierda de la figura 2; el Spaanse Kase, un complejo de edificios en terrazas que rodean un patio interior y las Kubuswoningen, una exploración sucesiva de 38 casas cubo en una cuadrícula de mayor escala, además de dos "súper cubos", el SúperKube Austral, desarrollado como una escuela de arquitectura, y el SúperKube Nothernmont, destinado a funciones comerciales (figura 2).

La idea principal era la de crear una comunidad urbana semejante a un pueblo en el interior de una gran ciudad, una zona de alta densidad urbana, donde se pudiera vivir en condiciones agradables; por lo tanto, elevó todo el conjunto de viviendas para liberar el suelo. De este modo, el espacio público se cuela en el interior del complejo, donde la organización de las casas da lugar a unas zonas abiertas con vegetación, que permiten una circulación por la zona de planta baja (Helena Ariza, 2013).

\section{La Casa Cubo: desarrollo y materialización}

Las estructuras cúbicas que componen el conjunto se encuentran giradas a 54,7 grados, de modo que el cubo se empuja ligeramente hacia adentro. Este se halla inclinado, aparentemente en equilibrio sobre su fuste, de modo que tres lados miran hacia el suelo y tres hacia el cielo, metafóricamente (figura 3).

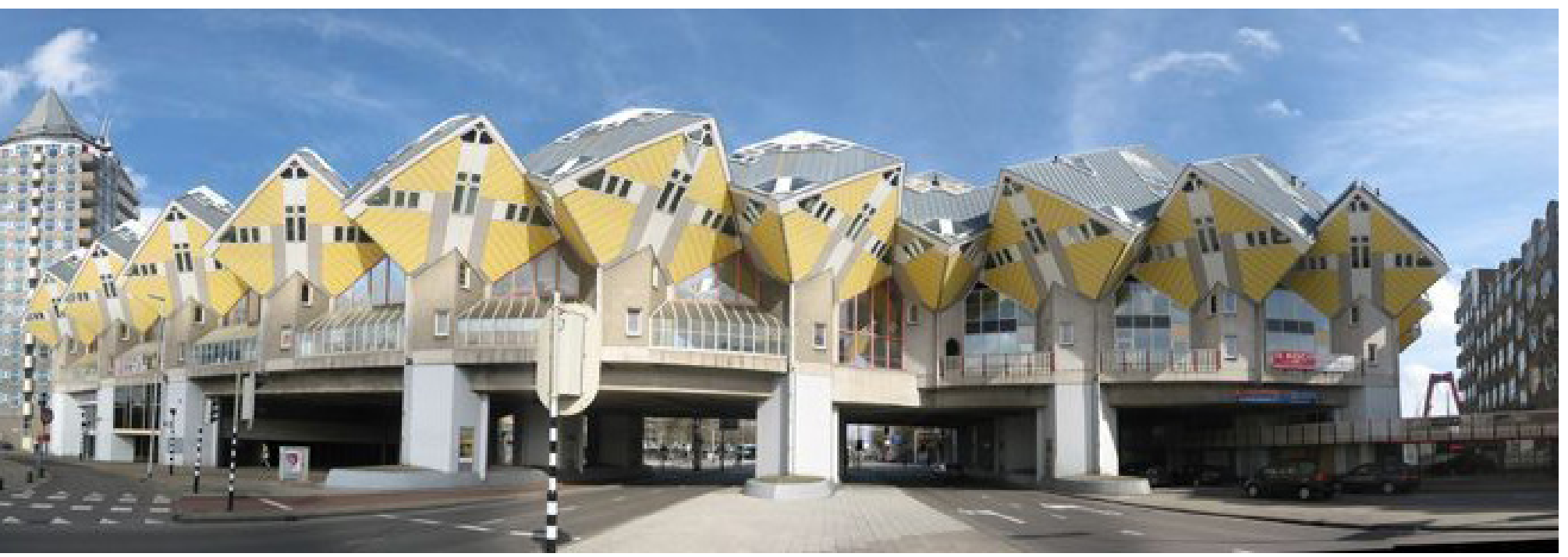

Figura 2. Kubuswoningen y SúperKubes. Fuente: https://jm3studio.com/casas-cubo-de-rotterdam/ 


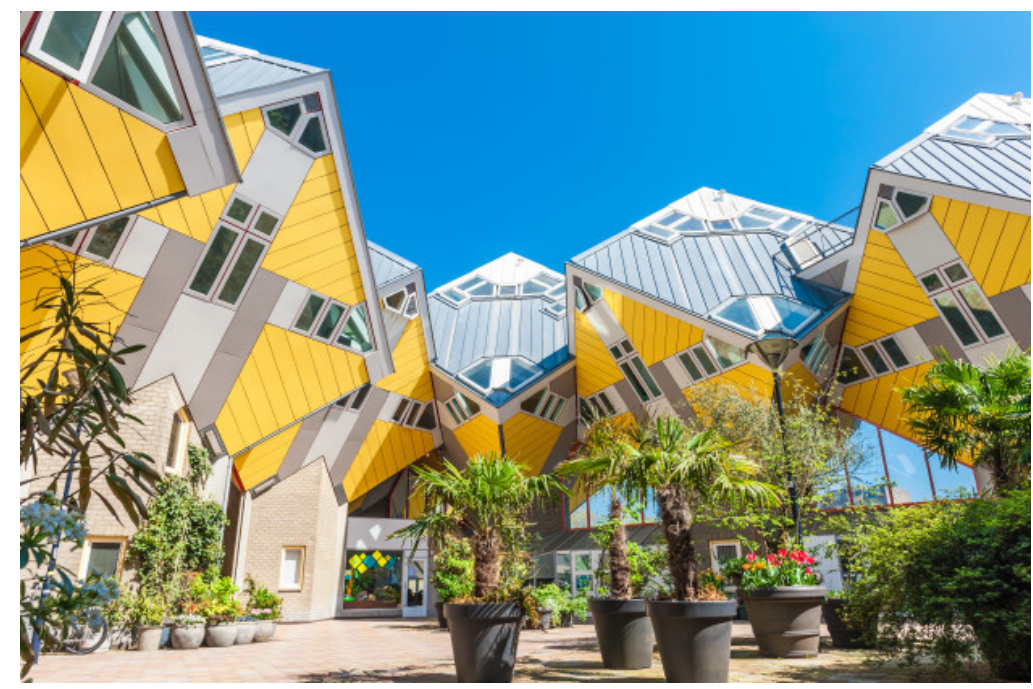

Figura 3. Ángulo de inclinación de las Casas Cubo. Fuente: www.wikiarquitectura.com

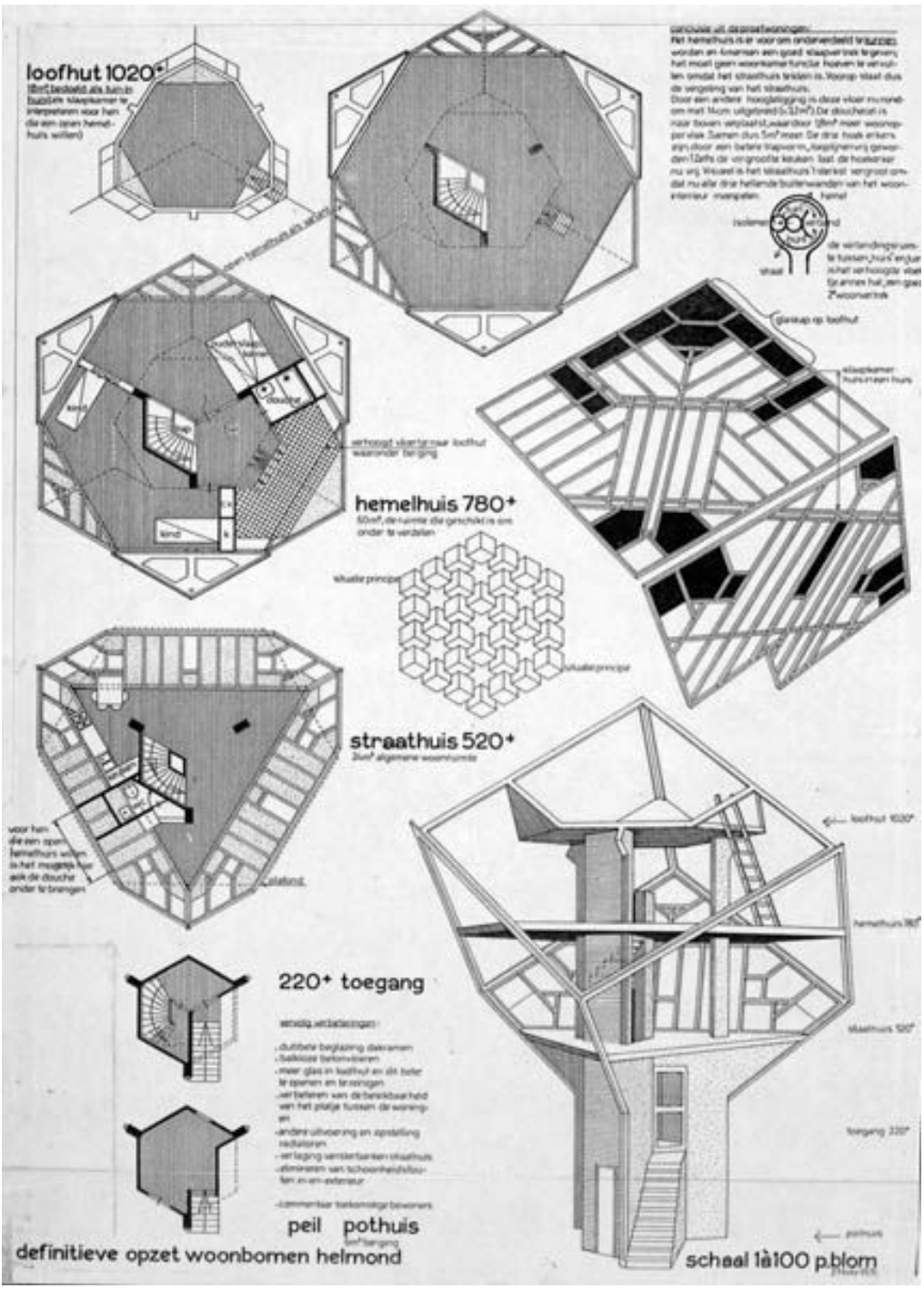

Figura 4. Plantas de niveles y axonométrica. Fuente: betonbabe.tumblr.com
Cada vivienda posee una superficie total de $106 \mathrm{~m}^{2}$, de los cuales el $25 \%$ es inhabitable debido a la inclinación de las paredes que crean espacios de difícil acceso, lo que es un llamado de atención en este trabajo: se puede observar que se hizo prevalecer la forma en detrimento de lo funcional con el desaprovechamiento del espacio. Cada Casa Cubo está dividida en tres niveles, como se puede observar en las figuras 4 y 5 : el primer nivel está destinado a la zona de estar, la cocina, ubicada en una esquina, con mobiliario hecho a medida, ya que los muebles tradicionales simplemente no encajan, y un área destinada al estudio o trabajo. Piet Blom llamó a este nivel el callejón, debido a que sus ventanas dirigidas hacia abajo permiten una conexión visual con lo que sucede a nivel de la calle. El nivel intermedio fue bautizado como sky-houseo, casa celestial, ya que alberga el área de dormir. Aquí se distribuyen los dormitorios, un pequeño salón y el cuarto de baño. Una plataforma con almacenamiento debajo y una escalera dan acceso al piso superior, el tabernáculo según Blom, que es un espacio de forma piramidal, con tres escotillas y dieciocho ventanas que dan una amplia vista a los alrededores y puede utilizarse según el deseo y necesidad de cada propietario.

Las casas tienen una altura de 22 metros (sin contar el fuste hexagonal), y cada uno de sus lados mide 7,5 metros. En las figuras 6, 7, 8 y 9 se pueden apreciar los espacios interiores detallados en cada nivel. El complejo fue creado teniendo en cuenta a la comunidad, ya que la zona peatonal que conecta los cubos mediante rampas incluye también un pequeño patio interno, oficinas y estudios.

\section{Fuste hexagonal y pisos interiores} la base del fuste tiene la forma de un hexágono, y está construido a partir de tres columnas de concreto de carga con paredes de ladrillo de hormigón de gas en el medio. Las 
escaleras a lo largo del lado conducen a la entrada (segundo piso). Tanto el fuste como las losas interiores fueron materializados con hormigón reforzado volcado in situ. El fuste tiene una altura de 5,20 metros, lo que da a la casa una altura total de 27,20 metros. Dependiendo del sector al que sirvan, cada una de las losas que actúan como separadoras de niveles poseen una forma particular. De esta manera, tenemos que la losa del primer nivel responde a la forma de un triángulo, la del segundo piso tiene forma hexagonal y en el nivel superior se repite la forma triangular. Esto responde a las particiones efectuadas dentro del cubo, resultado del análisis Ilevado a cabo por el arquitecto en el momento de realizar el diseño del prototipo de Casa Cubo (ver figura 10).

Estructura principal: cada cubo está conformado por un esqueleto de madera, sistema constructivo industrializado conocido como Ballon Frame, que se une a los bordes del piso de hormigón.

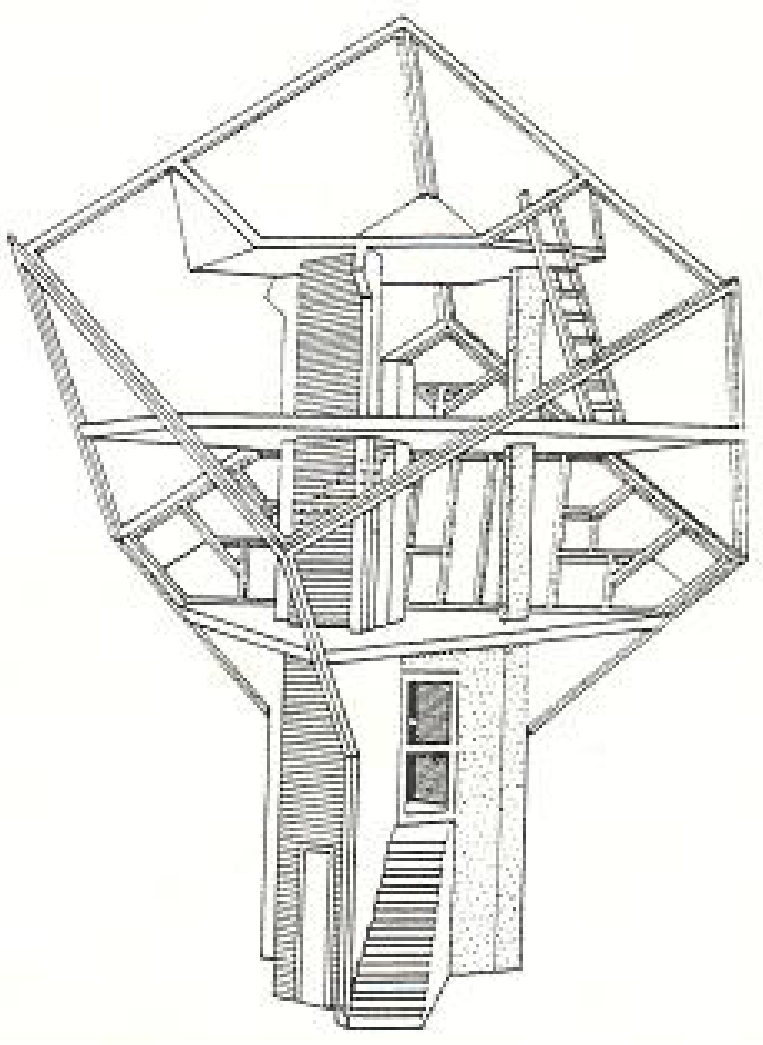

Figura 5. Axonométrica ampliada.

Fuente: betonbabe.tumblr.com
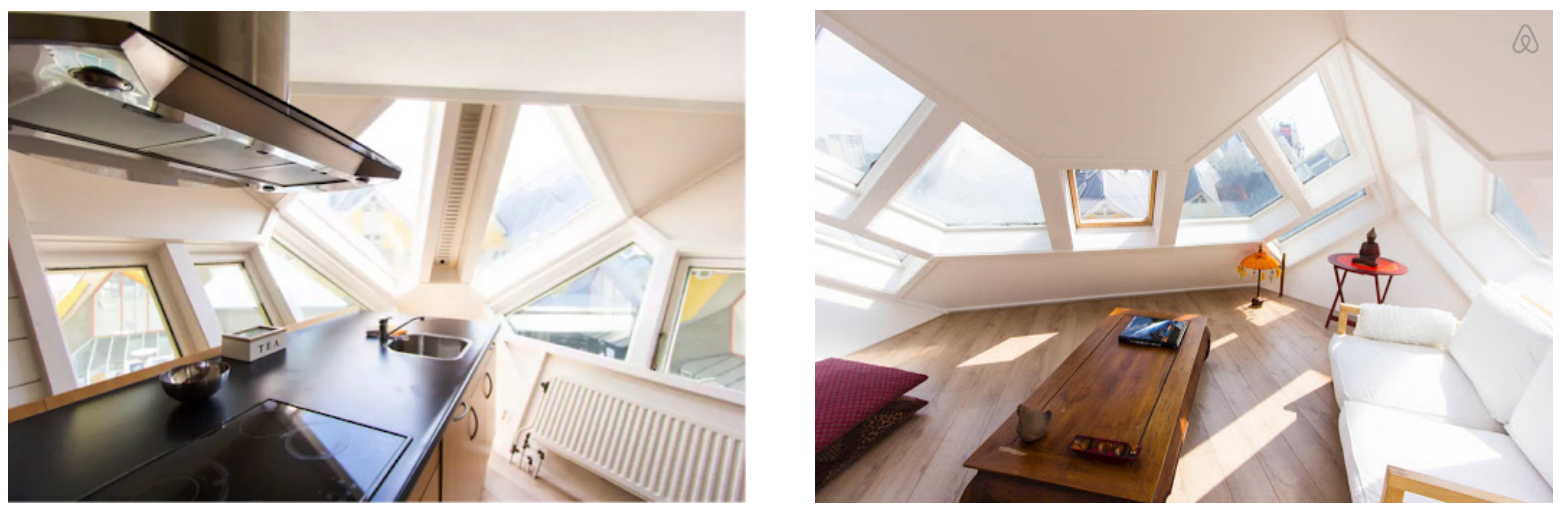

Figuras 6 y 7 (arriba). Cocina y sala de estar en el primer nivel. Fuente: www.pinterest.com
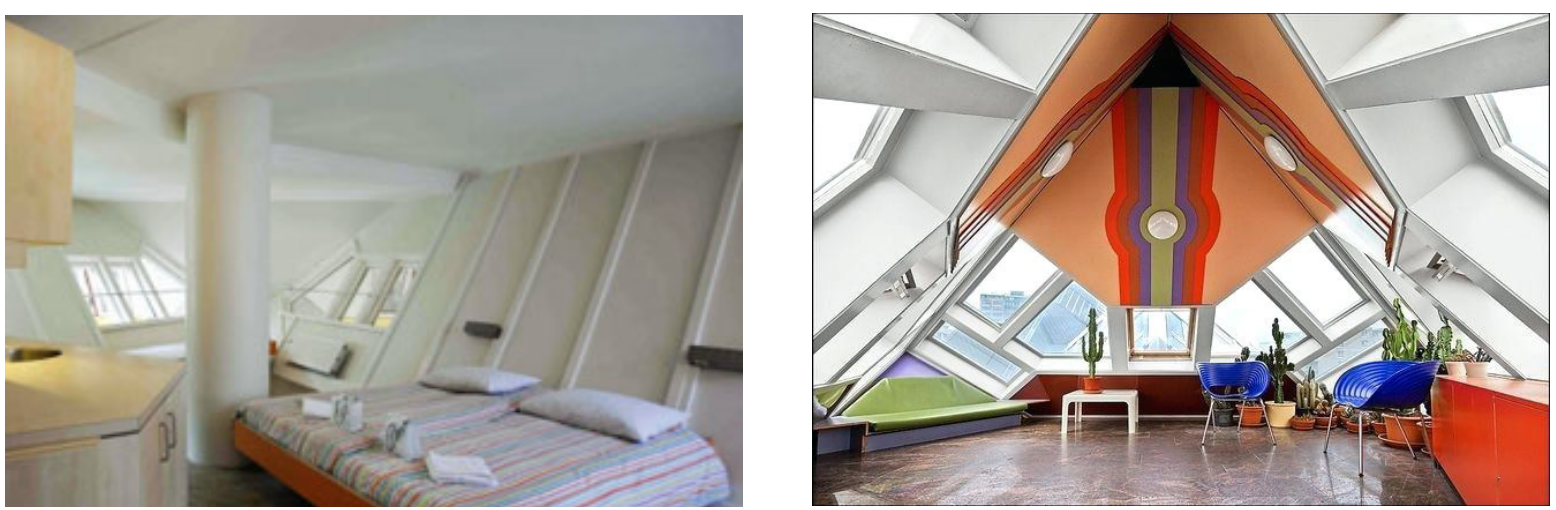

Figuras 8 y 9. Dormitorio principal (segundo nivel) y sala de juegos (tercer nivel). Fuente: www.pinterest.com 


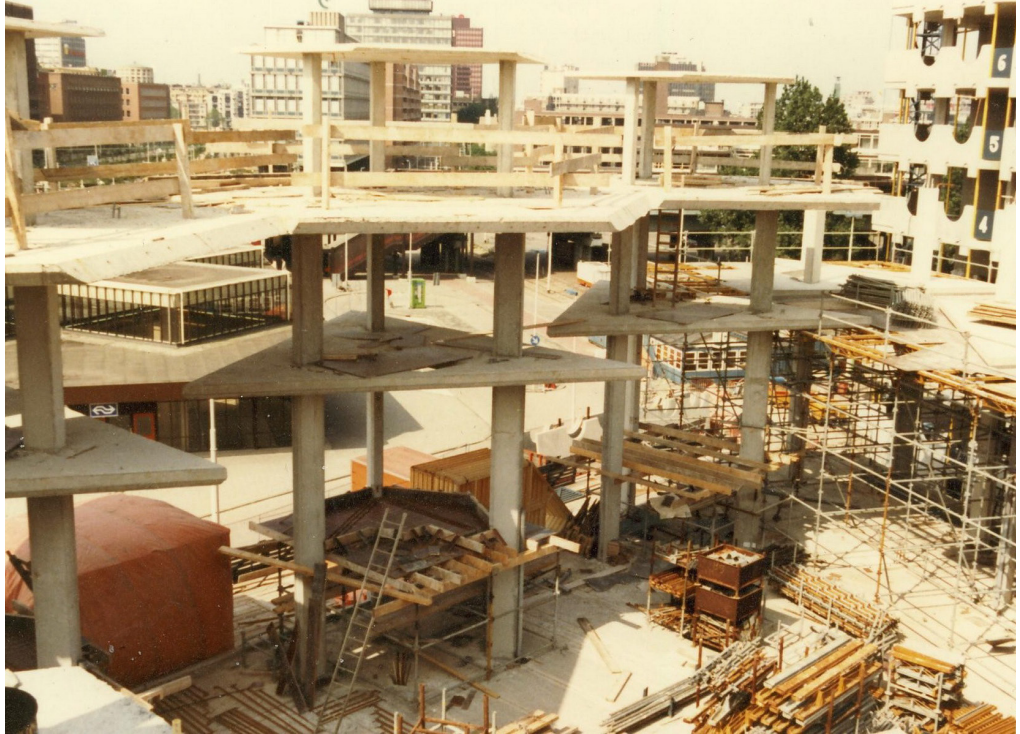

Figura 10. Materialización fuste. Fuente: architecturalvisit.com

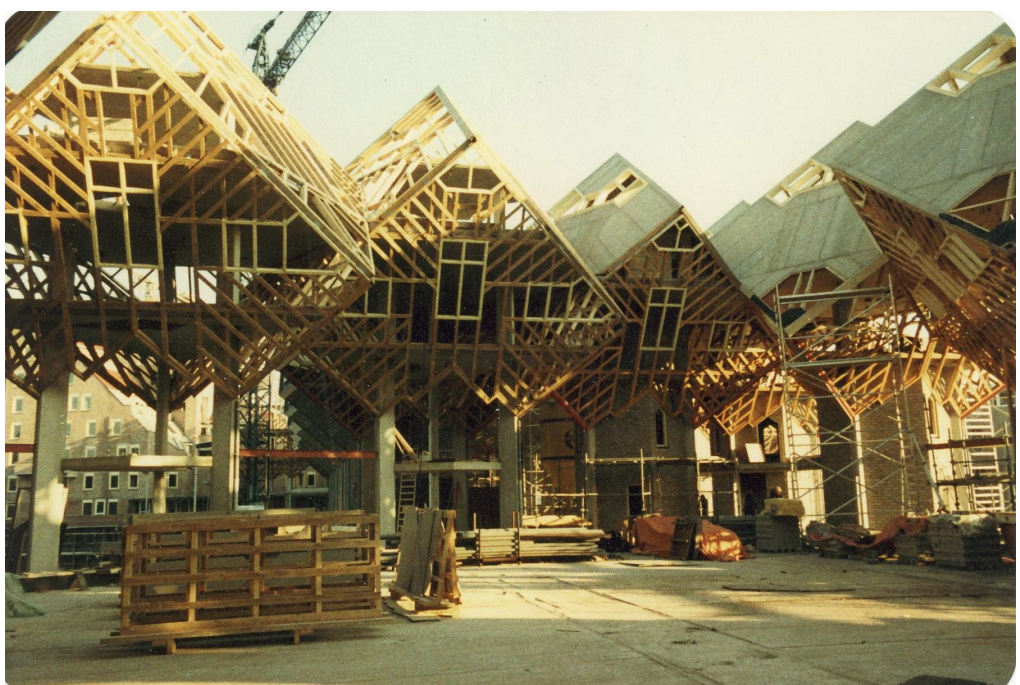

Figura 11. Estructura principal. Fuente: architecturalvisit.com
Tanto el exterior como el interior del esqueleto están cubiertos con paneles de fibrocemento de 18 $\mathrm{mm}$, atornillados y con lana de roca como aislante térmico en el medio (ver figura 11).

Todas las ventanas del cubo están constituidas con doble vidriado hermético y vidrio reforzado con malla de alambre donde es necesario. La primera capa de vidrio de las ventanas que miran hacia abajo es de $12 \mathrm{~mm}$ de espesor. La mayoría de las ventanas no son operables, para dotar de mayor aislación acústica al interior (en las figuras 12 y 13 se aprecian estas aberturas vistas desde el interior y exterior de la vivienda). Con las ventanas cerradas, casi no se oye ruido de tráfico, lo cual es fundamental para garantizar el confort interno, ya que gran parte de ellas dan hacia la arteria Blaak de siete carriles de circulación vehicular. Además, esto permite calentar la casa fácilmente. Gracias a la geometría y orientación de la casa cubo, las demás ventanas pueden abrirse y conseguir así la ventilación interior necesaria.

Entre los años 1997 y 1998 se le dio al edificio una nueva apariencia. Un techo de zinc con una capa de poliéster blanco sobre la estructura existente y una nueva capa de pintura en las superficies inferiores. Durantelos años
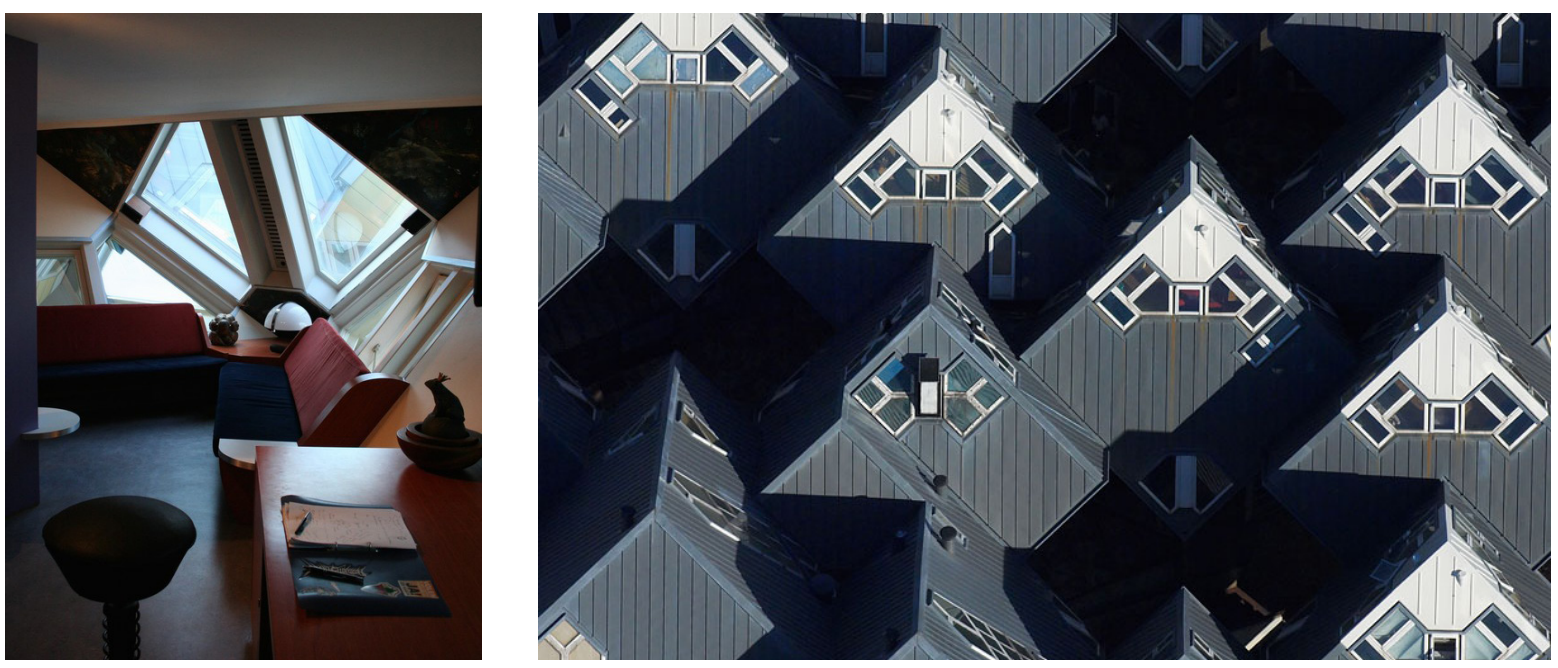

Figuras 12 y 13. Ventanas desde interior y exterior. Fuente: https://jm3studio.com/casas-cubo-de-rotterdam/ 
2000 y 2001, el paseo interior Overblaak fue restaurado, renovándose las escaleras, los adoquines y el alumbrado público (kubuswoning.nl/ woningen.html, 2019). Los habitantes le otorgaron al paseo una atmósfera más placentera y habitable colocando muchas macetas y plantas en su superficie (ver figura 14).

Para la segunda restauración, en 2015, se utilizaron andamios especialmente desarrollados que se izaron sobre las cimas de los cubos. Se cambiaron y aislaron las ventanas de los pisos superiores y las paredes fueron pintadas nuevamente. Podemos apreciar aquí cómo la construcción industrializada y racionalizada ya estaba presente desde la década de los 80, permitiendo acelerar los plazos de obra y otorgando mayor facilidad constructiva.

\section{Arquitectura y metáfora: viviendo como un techo urbano}

Viviendo como un techo urbano es el concepto en el cual Piet Blom basó la concepción de las Casas Cubo. El conjunto fue ideado como un bosque abstracto, donde cada vivienda representa un árbol y todas las casas juntas se asemejan a un bosque, impresión que el complejo daba a las personas, motivo por el cual se lo apodó Blaak Forest (Bosque Blaak).

Según Blom, la parte superior de cada casa, sostenida por un grueso núcleo como un tronco, representa un árbol abstracto que, conectado con su vecino, genera un gran bosque amarillo y manufacturado (figuras 15 y 16), idealizando la experiencia que supone vivir en los árboles y presentando una alternativa a la vida urbana en una gran ciudad. Esto requiere un poco de imaginación y adaptabilidad por parte de los residentes, y debe prosperar la creatividad, ya que los muebles estándar simplemente no encajan en este diseño, pero sin dudas ofrece una nueva y diferente posibilidad de vivir y valorar los espacios urbanos y la arquitectura.

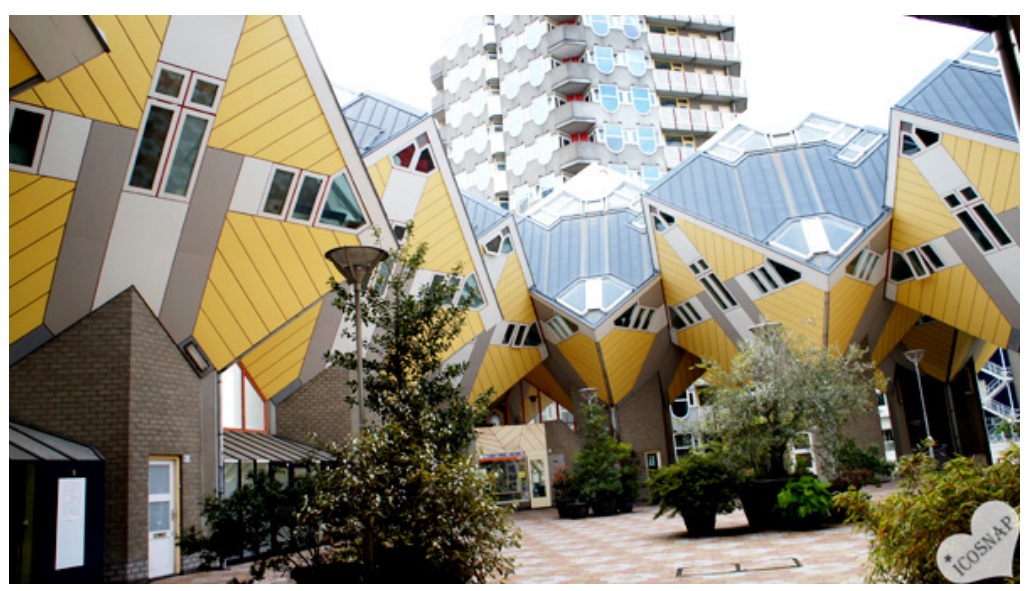

Figura 14. Paseo interior. Fuente: architecturalvisit.com

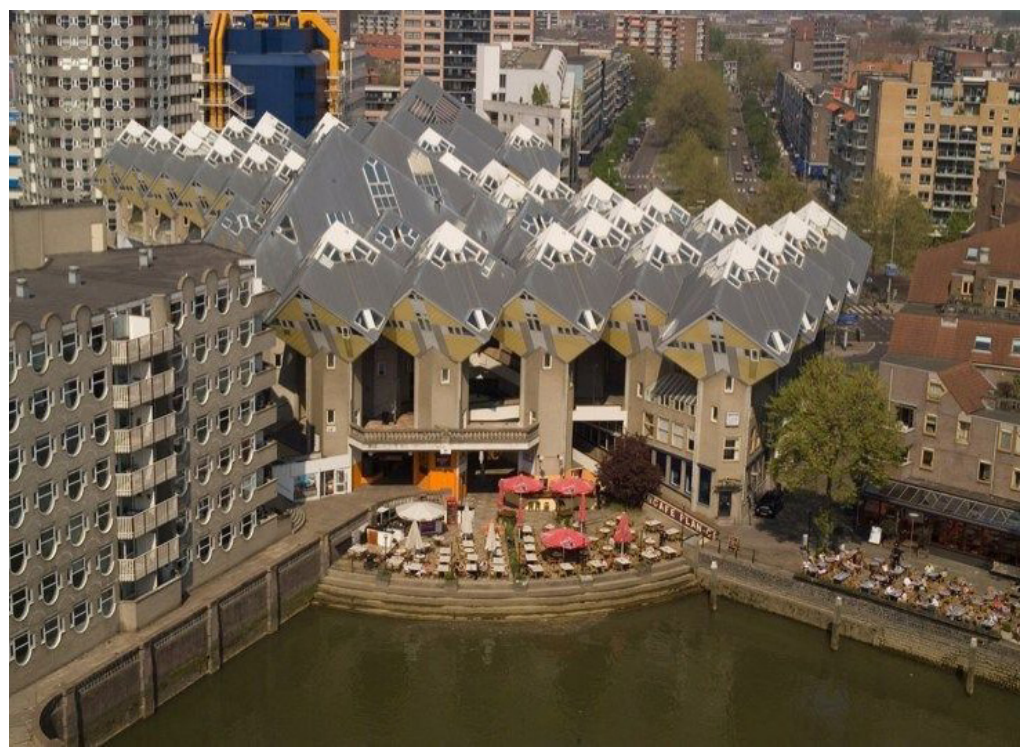

Figuras 15 y 16. Bosque urbano. Fuente: AD Classic Arch Daily

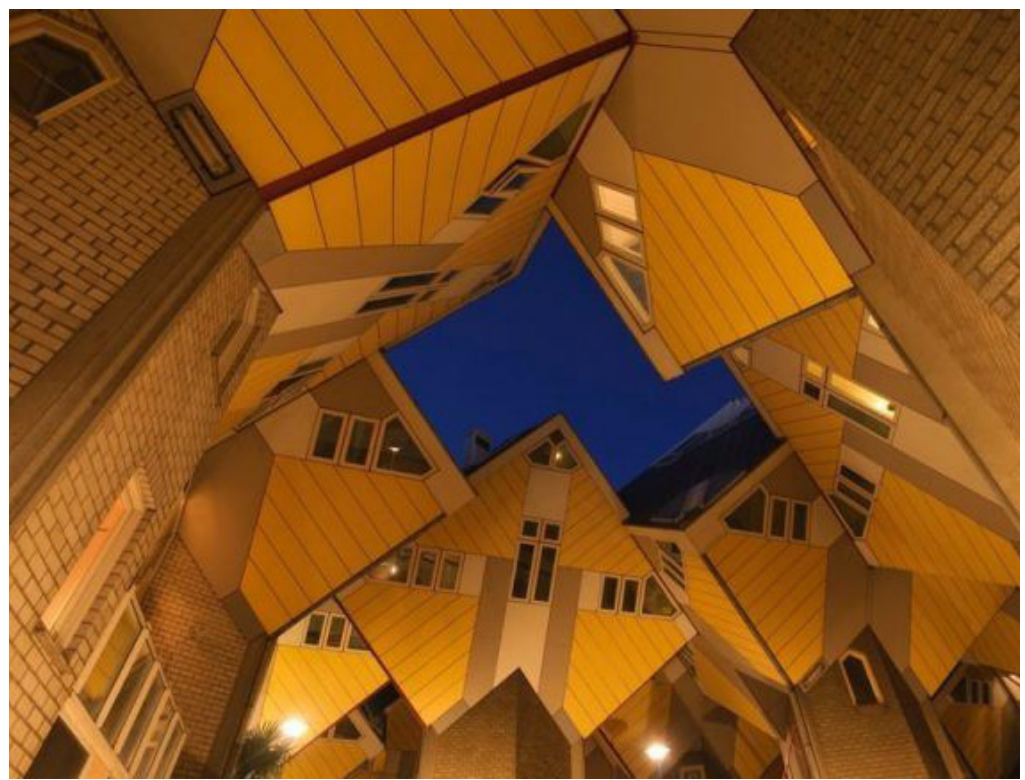




\section{Coordinación Modular: eficiencia en los procesos de diseño y construcción}

La coordinación modular, dentro de la Arquitectura Modular, es un sistema que simplifica y coordina las dimensiones de los elementos de construcción destinados a ser ensamblados, mediante medidas comunes y sus múltiplos, con el fin de lograr un máximo de eficiencia en los procesos de diseño y construcción de los edificios. Su utilidad para la industria de la construcción es muy grande, frente a la complejidad y variedad de los elementos constructivos, los cuales provienen de industrias o fábricas diferentes. Constituye una verdadera teoría del diseño, tanto de los componentes como del edificio mismo. Como consecuencia, la arquitectura va adquiriendo rasgos distintivos del diseño industrial, conciliando exigencias funcionales, técnicas y estéticas del producto, con medios y procesos de producción (Vedoya, 2016).

Con los términos "coordinación dimensional" o "correlación dimensional" se indica en la edificación un mecanismo de simplificación y conexión de las distintas magnitudes relativas y los objetos diversos, de distinta procedencia, que deben acoplarse entre sí en la fase del montaje, sin retoques ni ajustes. Esta premisa se consigue con el necesario complemento de algunas acciones normalizadoras (simplificación, unificación), que usualmente impulsan el desarrollo racional de la actividad de producción en serie de tipo industrial (Vedoya, 2016).

La coordinación modular utiliza tres herramientas básicas que le permiten lograr la compatibilidad en las tres instancias: diseño, fabricación y montaje de los componentes constructivos:

\section{- El Sistema Modular de Medidas} su adopción, como base de la normalización de los elementos de la construcción, es una condición fundamental para industrializar la producción, ya que permite coordinar las dimensiones de los elementos que constituyen el edificio, dimensiones que deberán tener una gran elasticidad de empleo y facilidad de producción en serie.

\section{- La Grilla Modular de Referencia} constituida por puntos, rectas y planos, a la que se referencian todos los componentes del sistema constructivo. La utilización de esta grilla abarca todo el proceso constructivo, desde el diseño del proyecto y sus partes, hasta el de la ejecución o "montaje" en obra.

- La Teoría de las Tolerancias y los Acoplamientos: es el margen de aceptación del error dimensional que presenta la medida efectiva del componente. Las medidas o dimensiones que maneja son: el Área Modular, Ia Discrepancia Modular Inferior, el Límite Modular Superior, la Medida Efectiva del Componente, la Tolerancia de Montaje, el Límite Modular Inferior y la Discrepancia Modular Superior.

La Coordinación Modular exige que todas las dimensiones de los componentes del edificio estén relacionadas entre sí, es decir, coordinadas, cuya unidad de medida y factor numérico es conocida con el nombre de módulo. El Módulo Básico (figura 17), se denomina con la letra $M$ y su valor internacional (normas IRAM) está normalizado en $10 \mathrm{~cm}$; por lo tanto, $10 \mathrm{~cm}$ equivalen a $1 \mathrm{M}$.

\section{MÓDULO BÁSICO}

$1 \mathrm{M}=1 \mathrm{dm}=10 \mathrm{~cm}$

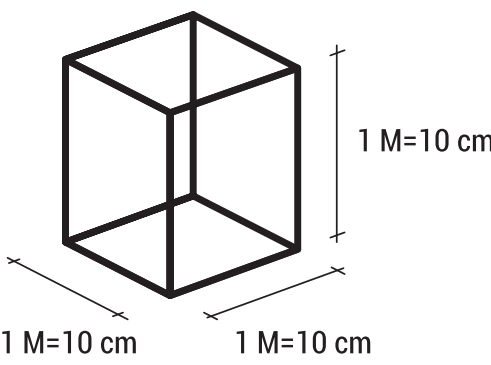

Figura 17. Módulo básico: $10 \mathrm{~cm}$. Fuente: publicación didáctica cátedra Construcciones II A

El módulo es el nexo dimensional entre el diseño y la construcción, que permite asegurar la disminución de la variedad de las dimensiones de los componentes constructivos y la correlación de las medidas entre ellos, su intercambiabilidad y aditividad con el máximo número de combinaciones posibles y la eliminación de desperdicios, cortes y ajustes ejecutados en obra. El módulo es aplicable en todas las etapas del proyecto, y constituye la base dimensional de donde derivan los tamaños de las distintas cuadrículas modulares: cuadriculado de planos, estructuras, de obra, de urbanismo, etc. (Vedoya, 2016). Las Casas Cubo presentan características concretas tratadas en el concepto de la Coordinación Modular, que es básica para la fabricación de los componentes de la construcción, sustentada en los principios de la industria: la racionalización del trabajo, la normalización constructiva y la tipificación productiva, como ya se mencionó en este trabajo.

Hemos podido observar cómo la modulación se puede utilizar en el diseño de proyectos individuales como a nivel urbano, donde el arquitecto Blom juega con las diferentes unidades funcionales, asignándoles a cada una un espacio concreto en la trama urbana por encima de las principales vías de circulación, liberando de esta manera el nivel peatonal y vehicular 
(figuras 18 y 19). Dota a su vez a cada casa de un carácter particular de modo que, si bien la tipología es repetitiva en todos los elementos que componen el conjunto, puede percibirse a cada una como un objeto único y contundente.

Vemos cómo Piet Blom aplica el método industrial de producción en el campo específico de la arquitectura, prestando especial atención a cada detalle y aspecto del proyecto, desde el diseño hasta su materialización constructiva. El uso de materiales normalizados (excepto en la estructura de sostén de los cubos), tanto en el proyecto original como en las sucesivas restauraciones realizadas a lo largo del tiempo, es un ejemplo claro de la implementación de los principios de la industrialización en todo el proceso creativo, proyectual y constructivo, cada uno ocupando un lugar específico en el espacio modular disponible.

\section{La Teoría de los Policubos como complemento de la Coordinación Modular}

Un policubo es una generalización tridimensional del concepto de poliminó, que consiste en un conjunto de módulos cuadrados unitarios unidos por sus lados. La teoría de policubos es una rama de las matemáticas que se ocupa de estudiar el comportamiento de unidades modulares cúbicas que unidas por sus caras configuran formas en e espacio tridimensional (Quezada Feijoó, 2012)

Las posibilidades de agrupación de los policubos son infinitas; cuanto mayor sea el número de cubos mayores serán las variantes que podamos obtener, de lo que resulta una gran variedad de módulos que conservan ortogonalidad entre sus caras y, dentro de la sencillez de sus formas, aportan riqueza volumétrica y modularidad, y se corresponden a formas aptas para su uso arquitectónico.
Para aplicar esta teoría, se tomaron cubos con diferentes medidas de lados: $2 \times 2 \times 2,3 \times 3 \times 3,4 \times 4 \times 4$; para implementarla en el proceso de diseño de una vivienda tipo, se escogió el cubo de $3 \times 3 \times 3$, ya que presenta las medidas estándares mínimas de una vivienda funcional. Una vez elegido el cubo de estas dimensiones, se analizaron las más importantes y conocidas disecciones que existen, y que son consideradas rompecabezas geométricos. Estos son: el Cubo de Conway, el Cubo 0 ' Berine, el Cubo Diabólico, el Cubo 7, el Cubo Soma, el Cubo de Nob, el Cubo de Coffin, el Cubo de Lola, el Cubo de Steinhaus y el Cubo Mikusisnki (figura 20).

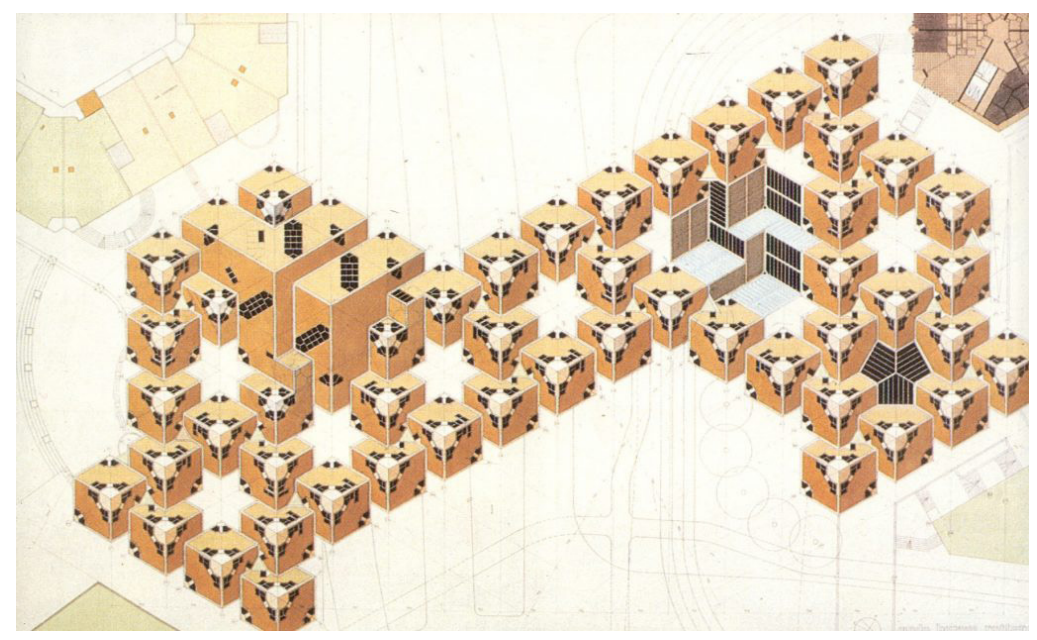

Figuras 18 y 19. Ubicación de las unidades en la trama urbana. Fuente: AD Classic Arch Daily

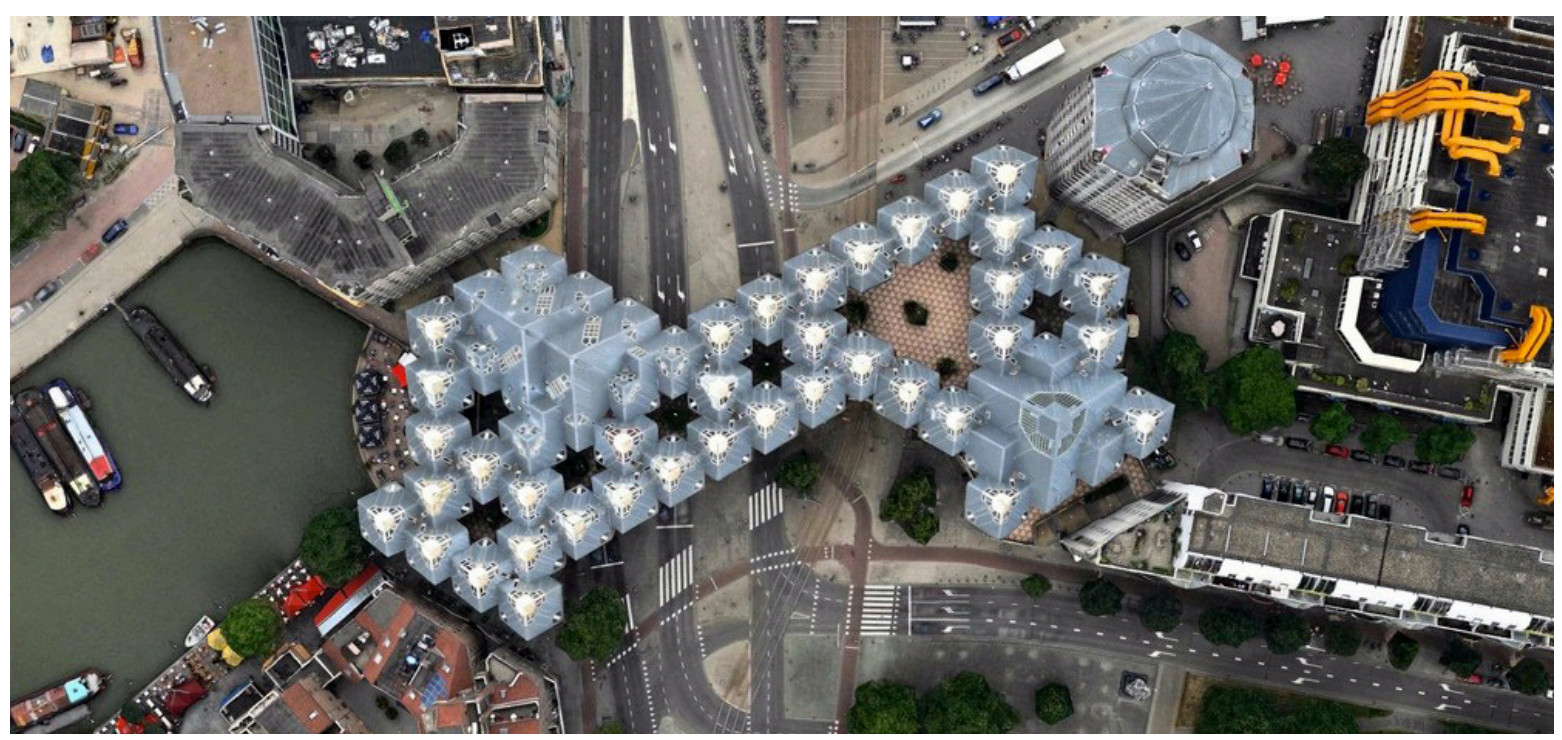




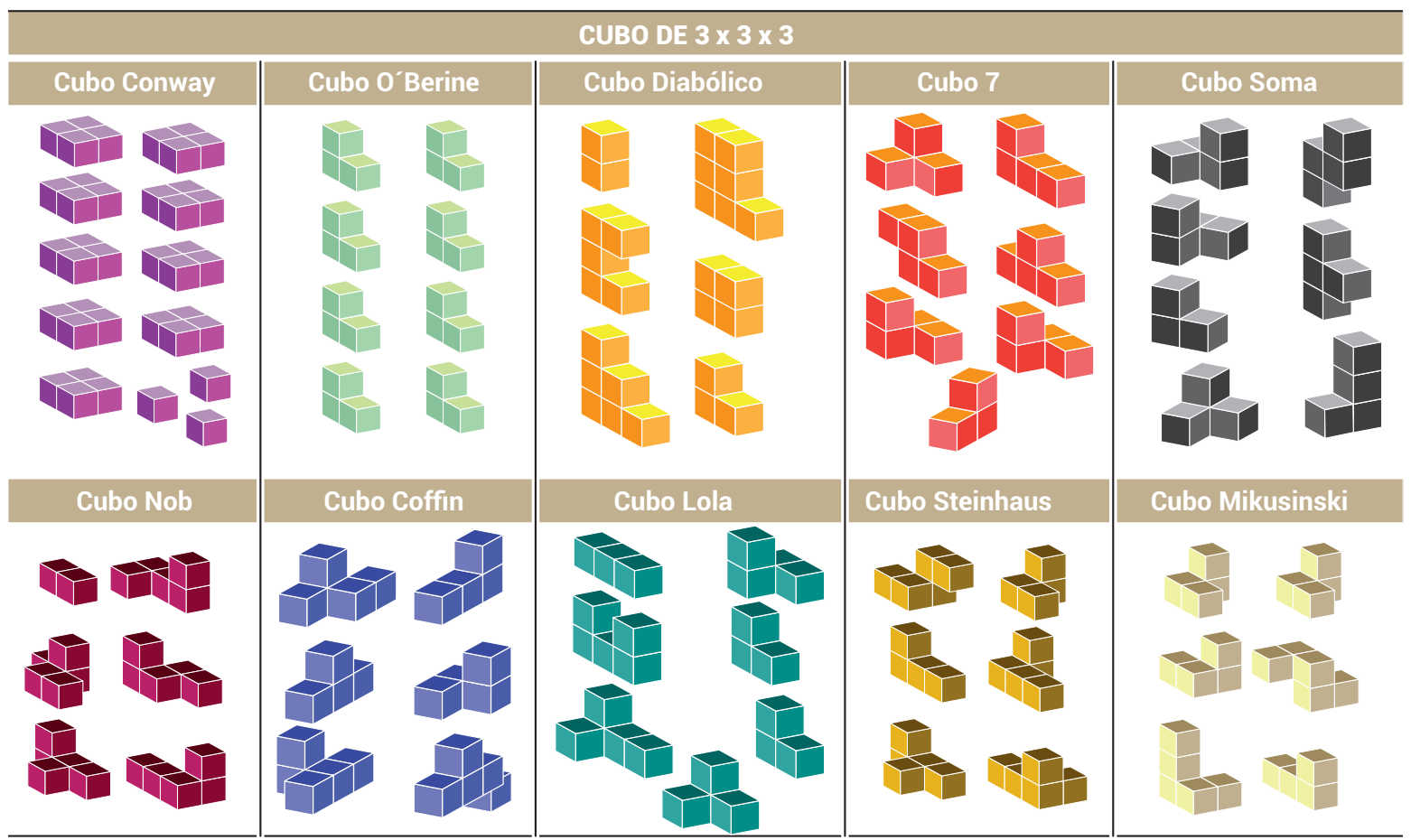

Figura 20. Disecciones de cubos de 3×3x3. Fuente: (Quezada Feijoó, 2012, Anexo 2)

De acuerdo con las formas que se obtuvieron de la combinación, por la libertad de agrupación que se les proporcionó, se analizó la construcción de cubos con policubos (cubos contenidos), tomando comoreferencia el caso particular del cubo de $3 \times 3 \times 3$, por el número adecuado de piezas policúbicas que contiene cada una de sus disecciones.

Al trabajar con la Teoría de los Policubos, cuyo elemento es el cubo, estamos definiendo un módulo de composición, ya que estas formas poseen un carácter modular interesante al configurar espacios tridimensionales macizos que se acoplan perfectamente por sus lados $y$, aun cuando se sustituya uno de ellos, el conjunto conserva la misma organización; de esta manera podemos observar claramente su correlación con los criterios de la Coordinación Modular y de qué forma puede influir en el proceso creativo. La utilización de la teoría de los policubos en el proceso de diseño permite reconocer que existen principios ordenadores que organizan la composición proyectada, y verificar que existen formas y espacios que satisfacen el desarrollo de las actividades que alberga el edificio proyectado.

Desde el punto de vista formal, es válida cualquier composición que resulte de adoptar un patrón y someterlo a alguna operatoria de simetría en el plano o en el espacio (incluyendo traslación, rotación, espejo, cambio de escala y todas sus combinaciones). En el caso de los policubos es una organización de cubos conectados por sus caras. Las piezas de un Cubo Soma, por ejemplo, constituyen patrones de repetición que pueden ser usados libremente para componer formas y asignarles una función (Quezada Feijoó, 2012).

Desde el punto de vista funcional existen principios de organización de las partes que albergan actividades, que responden a condicionantes y requerimientos arquitectónicos previamente definidos. Si partimos de un vocabulario básico de organización (central, lineal, radial, etc.) y de los principios ordenadores (ejes, simetría, jerarquía, ritmo, etc.), es posible asociar la oferta de formas con la función asignada a cada espacio, por lo cual es fundamental analizarlas previamente para adecuarlas al tipo de organización y sus características (Quezada Feijoó, 2012).

Si bien en la Teoría de los Policubos se utiliza una combinación de cubos, cuyo número varía de acuerdo con la cantidad de espacios funcionales requeridos en el programa arquitectónico para analizar y proyectar una vivienda unifamiliar, y teniendo en cuenta que cada una de las casas cubo se desarrolla proyectual y constructivamente dentro de un único cubo, creemos factible la aplicación de esta teoría a esta unidad de estudio, si la aplicamos al análisis del conjunto en general. De esta manera podemos observar cómo, partiendo de un cubo elegido con sus medidas específicas, el arquitecto va combinándolos mediante la yuxtaposición de sus caras, realizando particiones y adiciones de elementos (en este caso los troncos de hormigón que componen el acceso a las viviendas) y repeticiones sucesivas de las unidades, asignándoles diferentes funciones, sin modificar el carácter de los cubos ni a la armonía del conjunto en general. 

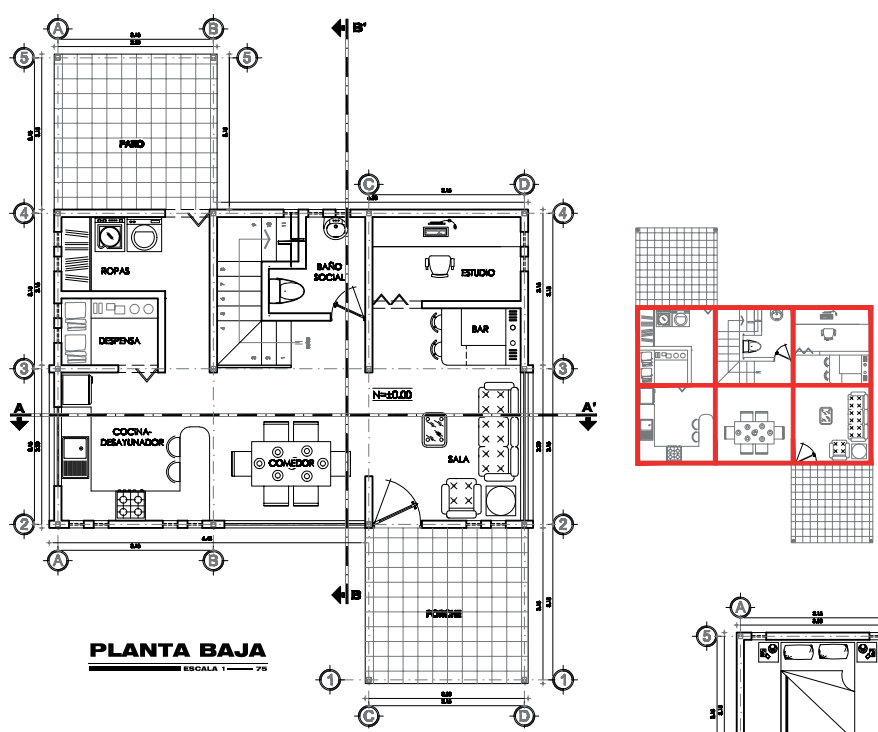

Figuras 21 y 22. Aplicación de la Teoría de los Policubos a proyecto de una vivienda. Fuente: Quezada Feijoó, 2012

En las figuras 21 y 22 podemos observar el análisis y la forma en que el autor Edmundo Quezada Feijoó aplica la Teoría de los Policubos en el proyecto de una vivienda; mientras que en la figura 23 vemos el diseño concebido por Piet Blom, al cual le establecemos algunos de los principios de dicha teoría, comprobando su adaptabilidad y correlación con la coordinación modular en toda su extensión espacial.
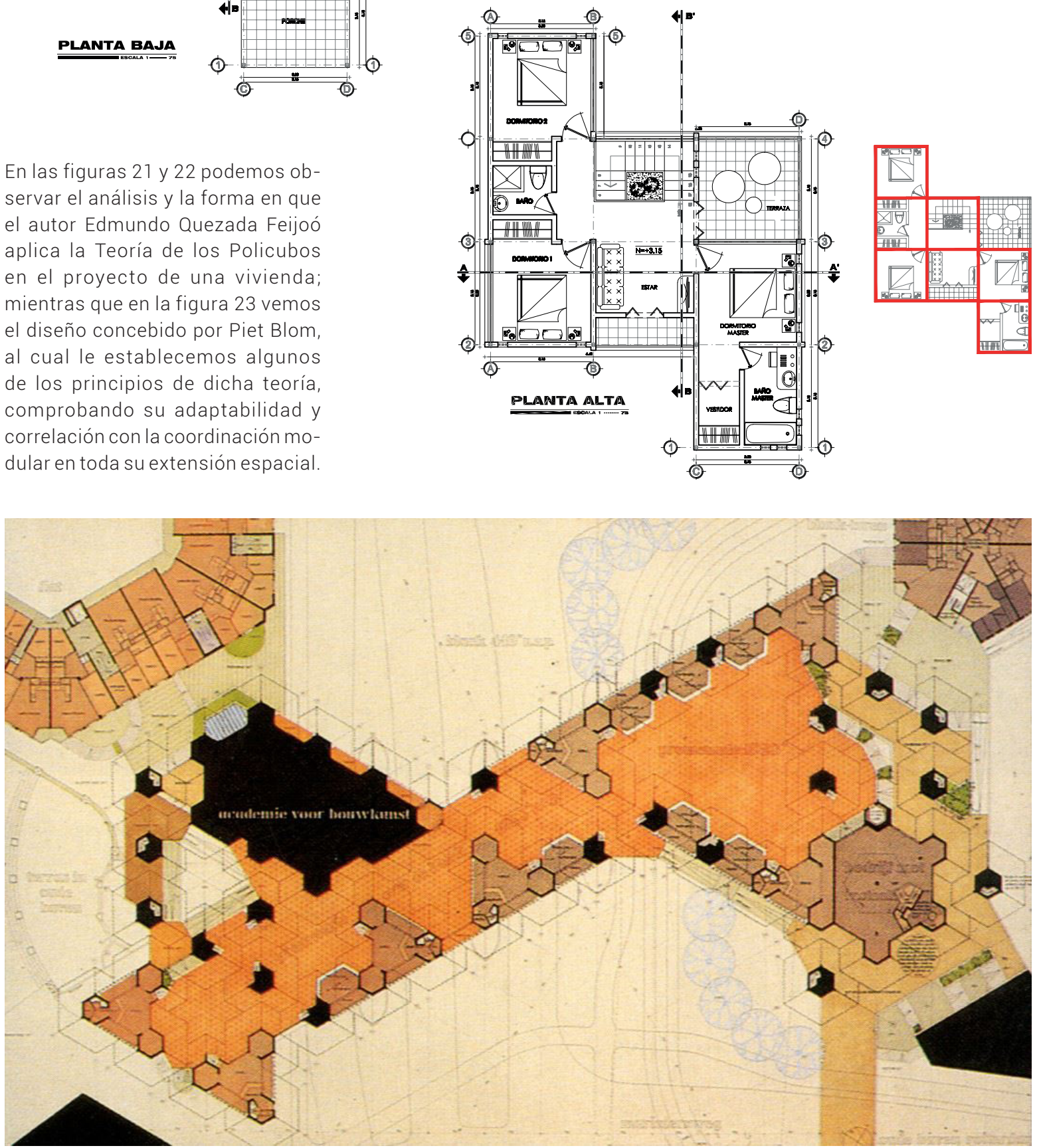

Figura 23. Aplicación de la Teoría de los Policubos al conjunto Kubuswoningen. Fuente: AD Classic Arch Daily 


\section{CONCLUSIONES Y REFLEXIONES FINALES}

El presente análisis nos permitió arribar a varias a conclusiones, todas de gran valor e importancia para el trabajo. En primer lugar, se pudo tomar contacto con una obra de arquitectura emblemática, que incluye a lo largo de todo su desarrollo creativo y constructivo los principios de la arquitectura industrializada, implementando soluciones tecnológicas no convencionales, que es justamente un punto fundamental en la temática académica de la cátedra Construcciones II A, donde además se desarrolla esta investigación y es la asignatura beneficiaria directa de los resultados del trabajo. A su vez, se puede comprobar cómo diferentes conceptos que parecen aislados, como la Coordinación Modular, la Teoría de los Policubos y los elementos constructivos racionalizados, encuentran una correlación, conectándose de una manera muy íntima a través de sus principios y criterios particulares, que en un punto comparten.

Este análisis permite que nos apartemos de las resoluciones tradicionales, para descubrir y explorar un nuevo mundo de posibilidades proyectuales, técnicas y creativas y arribar a resultados más satisfactorios e innovadores gracias a sus múltiples ventajas, referidas a sus movimientos de intercambiabilidad y aditividad. Así se logra un máximo de combinaciones posibles y una reducción de variables dimensionales, lo que significa una gran disminución de desperdicio de materiales y tiempos de ejecución, que se traduce en una mayor rentabilidad y beneficios económicos.

Asimismo, el objeto de estudio analizado, las Kubuswoningen, constituye un claro ejemplo de la factibilidad de incorporar nuevas e interesantes propuestas creativas, aplicando en su desarrollo innovaciones tecno- lógicas para lograr un producto funcional, armonioso, llamativo y de calidad, y derribar los mitos de que los objetos arquitectónicos realizados con sistemas constructivos no convencionales son repetitivos, insulsos y proclives a la desconfianza en cuanto a su durabilidad, estabilidad y buen funcionamiento. Esto da la posibilidad de fortalecer y ampliar su desarrollo y difusión a nivel local y propiciar la evolución constante de esta grata tarea de hacer Arquitectura.

\section{BIBLIOGRAFIA}

ARIZA, Helena (2013). KUBUSWONINGEN: Excentricidad elevada al cubo. Disponible en http://www. architecturalvisits.com.

QUEZADA FEIJOÓ, E. D. (2012). Arquitectura modular basada en la teoría de policubos. Trabajo de fin de titulación, Universidad Técnica particular de Loja - Loja (Ecuador). Director: Arq. Xavier Burneo. Recuperado de https://1library.co/ document/ozle86q4-arquitecturamodular-basada-en-la-teoria-delos-policubos.html

VEDOYA, D. E. (2016). La Coordinación Modular (publicación didáctica). Cátedra de Construcciones II: Facultad de Arquitectura y Urbanismo (FAU), Universidad Nacional del Nordeste (UNNE).

\section{PÁGINAS WEB CONSULTADAS}

www.jm3studio.com/casas-cubode-Rotterdam

http://www.kubuswoning.nl/

woningen.html

www.wikiarquitectura.com

https://ar.pinterest.com/ pin/78813062205542155/

https://nl.wikipedia.org/wiki/ Kubuswoningen_(Rotterdam) http://circarq.wordpress.com http://www.architecturalvisits.com https://www.archdaily.com/ architecture-classics https://planergo.com/es/sitios/

Casas-cubo-de-Helmond/

www. betonbabe.tumblr.com 
